\title{
33
}

\section{Assessing the Quality of Requirements Engineering Products}

\author{
R E M Champion \\ Department of Information Systems, City Polytechnic of Hong Kong, Kowloon, Hong Kong
}

\begin{abstract}
The specification of user requirements for software systems provides the foundation for the subsequent development work. This paper examines the criteria for assessing the quality of the products of requirements engineering, and the implications of these criteria on the structure and content of requirements models. In particular, it is argued that the criteria of traceability and and its impact on the maintainability of the final product imply the need for more comprehensive models of requirements statements.
\end{abstract}

Keyword Codes: D.2.1, D.2.4, D.2.9

Keywords: Requirements Engineering; Conceptual Modelling; Quality Assurance

\section{INTRODUCTION}

This paper examines the products of the requirements engineering (RE) phase of software systems development, specifically in terms of the quality criteria which are being proposed for these products [1-2]. The intention is to demonstrate that the traditional product of the process is not capable of supporting current demands; and that to satisfy existing quality criteria, new products need to be explicitly described.

The paper first outlines the process of RE in terms of its inputs, transformations and outputs. The nature of the output from the RE process will vary according to the method and models used, and is currently the subject of much debate [3]. Consequently, this paper describes the products in terms of a set of quality criteria, compiled from the literature, which effectively differentiates between good and bad RE products. The quality criteria are then classified according to the purpose of the RE product being measured, and this classification is compared with needs of the various types of personnel who interact with the product. The comparison is used to propose a richer modelling environment for the RE process, which facilitates the representation of requirements knowledge in different models, depending on the aspects of quality which should be addressed by each component.

\section{THE PROCESS OF REQUIREMENTS ENGINEERING}

Requirements engincering is traditionally viewed as that process which transforms the needs and wishes of potential users of the target computerised system, usually incomplete, and expressed in informal terms, into a complete, precise, and consistent software requirements specification (SRS). The primary process is accepted as being an iteration of the three tasks: elicitation, modelling and validation [4-5]. The cycle of iteration continues until the model is considered to contain sufficient information for the design process to begin and, in practice may continue beyond this point, either as requirements change or designers seek further information [6].

The customers are involved both with the validation of the evolving model and with the review of the SRS as the basis of a contract. The elicitation task may utilise a variety of approaches to gather information for the modelling task, but the sources of the information are limited to people and documents [7]. One special form of documentation is the 'requirements 
document', which can be used to express an initial version of customers' requirements using natural language, as a starting point for the modelling task [8].

In order to specify the content of the information which is acquired by the analysis phase, it becomes necessary to examine the context in which the people and documents exist. The issues addressed by a full analysis of an enterprise's information system requirements are described in [910], but include the organisation, the business processes, the mission and environment. None of these categories of information can be considered as fixed for an enterprise. While much work is being done to facilitate the consensus formation [11], the emphasis is still on the construction of a single view, albeit a view which is acceptable to all parties concerned. While a single view of the required system is essential for the developers, it is less so for system designers, and is a positive disadvantage to other potential users of the RE products who need to understand the complexities of potential futures for the enterprise.

\section{EVALUATING THE PRODUCTS OF REQUIREMENTS ENGINEERING}

While the products of requirements engineering are generally viewed as consisting of a single artefact, the SRS, there is no standard for the content of this product. Attempts to differentiate between good and bad products have resulted in the formation of quality criteria [2], but these criteria are specified, applied and evaluated with the assumption that a single product is being measured. Consequently, products with different functions are being compared using the same criteria; or, where a single product is precisely defined, that product is subject to the application of all quality criteria, which are often contradictory.

\subsection{Fact Elicitation}

At the initial stage of analysis, statements concerning any of the aspects of the enterprise may be elicited from the customers, either as the results of observation and interviews, or by the analysis of the initial requirements document. Since the facts may legitimately represent different viewpoints of these different aspects, the only quality criteria which can be realistically applied to the product of this stage are:

(a) Traced; the source of the fact is recorded; (b) Traceable; the inclusion of the fact in any subsequent analysis model can be recorded; and (c) Verifiable; it is possible to determine the truth of the fact from its statement.

The last attribute allows for the removal of any incorrect facts elicited from the customers; no judgements can yet be made about ambiguities or inconsistencies.

\subsection{Modelling for System Developers}

The traditional end product of the RE process should provide the development team with sufficient information for the design and implementation of an automated information system. Hence, quality criteria for this product can be restricted to:

(a) Complete; everything that the IS is intended to do is included in the product; (b) Consistent; no part of the product conflicts with any other part; (c) Correct; all components of the product describe a required element of the system's behaviour; (d) Minimal; the product should not overconstrain system design; (e) Traced; elements of the product can be traced back to intermediate analysis products; (f) Traceable; elements of the product can be traced forward to design products; and $(\mathrm{g})$ Unambiguous; every element of the product has only one interpretation.

The first two products described above represent the start and end points for the RE process. While the aim of this paper is not to define a process model for RE, it is also necessary to consider intermediate products and their role.

\subsection{Intermediate Modelling}

The facts obtained from the customers during elicitation may contain knowledge concerning any aspect of the enterprise being analysed, and in any of the modes described above. In order 
to organise this information and be able to reason about the implications for the required system, it is necessary for the analysts to construct models of these various issues.

(a) Appropriate; elements of the product describe only features of the issue addressed; (b) Expressive; it is possible to describe any aspect of the issue addressed; (c) Inconsistency tolerant; it is possible that the product may contain legitimate inconsistencies, within different viewpoints; (d) Incompleteness tolerant; the product need only be as complete as the analysts decide; (e) Traced; elements of the product can be traced back to other intermediate analysis products, or directly to facts from the elicitation phase; and (f) Traceable; elements of the product can be traced forward to the final development product, or to other intermediate products.

Traced and traceable now describe a network of links between the initial and final products, through the intermediate models used by the analysts. The quality attribute 'modifiable' can be used to measure the quality of these links rather than of a particular product.

\subsection{Modelling for Validation of Requirements Products}

Validation of any of the products of RE, by definition, will involve the customers of the system interacting with the analysts. The aim of the interaction is to satisfy the customers that the evolving system description matches their requirements. Two methods of validation exist; either the product being validated is presented to the users for direct evaluation, or the product may be animated or executed to demonstrate aspects of the external behaviour of the target system. The first case adds 'understandable' to the list of quality attributes for the product being validated. For the product to be understandable by non-computer specialists, it may well be necessary to compromise some of the other quality attributes. In the second case, it is assumed that a separate product is involved, allowing the following product-specific quality criteria to be specified.

(a) Complete; everything that the product is expected to demonstrate is included; (b) Consistent; no elements of the product contradict any others; (c) Unambiguous; every element of the product has only one interpretation; and (d) Formal; the notation of the product permits a mechanistic analysis of the content.

The final product may be considered to result from the combination of the products described above, and the way in which the combined products facilitate changes to the requirements for the target IS, either during development or subsequently.

\subsection{Modelling for Maintenance and Reuse}

The product from this stage may be used by customers, analysts and developers, as well as maintainers. Quality of the product must be measured in terms of the ability of the users to determine the nature and consequences of altering any aspect of an enterprise modelled during the $\mathrm{RE}$ process, including the information systems. Since change requests may originate at any level of the enterprise, from its mission and objectives, to the tasks performed by individuals, the need for traceability becomes paramount. The following criteria should be used to measure the quality of the combined product of RE.

(a) Annotated; it should be possible to relate the necessity and stability of requirements to aspects of the intermediate models; (b) Expressive; everything that needs to be considered in evaluating changes is included in the product; (c) Modifiable; the structure of the product allows for alterations of individual components; and (d) Traceable; each component of the product is linked to related components, including 'derived from' relations.

\section{CONCLUSIONS}

The product description, contained in the SRS, forms only a part of the products of RE, and it is this part which requires consistency, completeness and preciseness. Other products, because of their failure to meet these 'quality' criteria have generally been treated as intermediate, 'throwaway' artefacts, useful only in their contribution to the construction of the SRS. However, such an SRS is only of direct use to the system developers, while other criteria for quality, from other user 
groups are difficult to match against this single product. The preceding sections have clearly shown the differentiation between the products required for each phase of the process, and the user groups involved at each phase. The advantage of this approach is that products can be evaluated according to criteria which specifically measure the quality required by the phase being addressed.

Finally, the languages and models used for each of the products should reflect the quality attributes addressed. This implies that a single notation may not be sufficient for all products. Consequently, the maintenance product, which involves the combination of other products, may require more sophisticated facilities than currently exist. The linking of concepts represented in different notations, so as to allow tracing of the development, requires further investigation.

\section{REFERENCES}

1. Davis, A. Software Requirements: Objects, Functions, and States; Prentice Hall, Englewood Cliff, NJ, 1993.

2. Lindland, O. Sindre, G. \& Solvberg, A. Understanding Quality in Conceptual Modeling; IEEE Software, March 1994.

3. Lubars, M. Potts, C \& Richter, C. A Review of the State of the Practice in Requirements Modeling; Proc. Int. Symposium on Requirements Engineering, IEEE C. S. Press 1993.

4. Armenise, P. Bandinelli, S. Ghezzi, C Morzenti, A. Software Processes Representation Languages: Survey and Assessment; Proc. Int. Conf; SEKE'92, Capri, Italy, June 1992.

5. Loucopoulos, P. and Champion, R. Concept Acquisition and Analysis in Requirements Specifications, Software Engineering Journal, Vol 5, No. 2, March 1990.

6. Harker, S. \& Eason, K. The Change and Evolution of Requirements as a Challenge to the Practice of Software Engineering; Proc. Int. Symp. on Reqs Eng; IEEE C. S. Press 1993.

7. Goguen, J. \& Linde, C. Techniques for Requirements Elicitation; Proc. International Symposium on Requirements Engineering, IEEE C. S. Press 1993.

8. Kliem, R. Back to Basics: Developing A Good Requirements Document, Journal of Systems Management, October 1992.

9. French, J. The Business Knowledge Investment; Yourdon Press, Englewood Cliffs, New Jersey, 1990.

10. Mills A, Murgatroyd S, Organisational Rules: A Framework for Understanding Organisational Action, OU Press, 1991.

11. Easterbrook, S. Domain Modelling with Hierarchies of Alternative Viewpoints; Proc. International Symposium on Requirements Engineering, IEEE C. S. Press 1993. 